\title{
ADULT B LYMPHOBLASTIC LEUKEMIA WITH A NOVEL DE NOVO CHROMOSOMAL TRANSLOCATION [der(9)t(9;12)(p24;q12),-12]: A CASE REPORT
}

\author{
Gadhia $\mathrm{PK}^{1},{ }^{*}$, Shastri $\mathrm{GD}^{2}$, Shastri $\mathrm{EG}^{2}$
}

*Corresponding Author: Professor Pankaj K. Gadhia, Department of Biosciences, Veer Narmad South Gujarat University, Surat, 395009 Gujarat, India; Mobile: +91-9825163395; E-mail: genecare3@gmail.com

\begin{abstract}
Patients suffering from adult acute lymphoblastic leukemia (ALL) are very ill and present most commonly with shortness of breath during physical activity, a pale complexion from anemia, a very low platelet count, the appearance of pinhead-sized red spots under the skin called petechiae, or prolonged bleeding from minor cuts and discomfort in the bones and joints. Fever in the absence of an obvious cause is common. Leukemic lymphoblasts may accumulate in the lymphatic system, and lymph nodes can be enlarged. We report a young adult male with a novel translocation in ALL, who had vague (uncertain) minimal symptoms and splenomegaly. The patient had a unique cytogenetic abnormality: 45,XY,der(9)t $(9 ; 12)(\mathrm{p} 24 ; \mathrm{q} 12),-12$, which has not been previously described in ALL. This is categorized as a poor risk due to his failure to achieve complete remission after induction chemotherapy.
\end{abstract}

Keywords: Acute lymphoblastic leukemia (ALL); Chromosome; Karyotype.

\section{INTRODUCTION}

Demonstrable cytogenetic abnormalities are observed in 70.0-90.0\% of cases of acute lymphoblastic leukemia (ALL). Cytogenetic abnormali-

\footnotetext{
${ }^{1}$ Department of Biosciences, Veer Narmad South Gujarat University Surat, Gujarat, India

${ }^{2} \mathrm{GeNe}$ CaRe Genetic Diagnostic Laboratory, Surat, Gujarat, India
}

ties such as $6 \mathrm{q}$ - and $9 \mathrm{p}$ - are associated with both B- and T-lineage ALL, while others are confined to one lineage or are associated with a specific immunophenotype within a lineage. Translocations with breakpoints involving immunoglobulin genes (heavy chain, $\kappa$ or $\lambda$ ) are generally B-lineage and those involving $T C R$ genes are largely confined to T-lineage ALL [1]. Hyperdiploidy is commonly associated with B-lineage ALL and is rare in T-lineage ALL. Precursor B-lympho-blastic leukemia/lymphoma is a lymphoproliferative disorder of immature B-cells with blastic morphology, which often express CD10, CD34, and TdT (clusters of differentiation). A number of clinical, phenotypic, and genetic features are prognostically significant in B-ALL. The major prognostic factors include age, white blood cell (WBC) count, time of response to treatment, chromosomal aberrations, minimal residual disease (MRD) and drug resistance. A better prognosis is associated with age of $<30$ years, WBC count $<30000, t(10 ; 14)$ and complete remission within 4 weeks. Factors associated with poor prognosis include elevated total WBC count $(>50.0$ $\left.\times 10^{9} / \mathrm{L}\right), \mathrm{t}(9 ; 22), \mathrm{t}(4 ; 11), \mathrm{t}(1 ; 19)$, hypodiploidy, -7 , +8 , expression of myeloid antigens such as CD13, CD33 and persistent MRD.

Case Description. A 36 - year old male presented with a history of aches and pains over his whole body, especially marked in joints, lower legs and both hip joints lasting for 1.5 month. He also suffered from fatigue and fever. There was no history of allergies, skin rash, cough, urinary and bowel 
complaints. He is self-employed in the textile business, does not smoke, does not have diabetes, no hypertension and is not an alcoholic. At the time of his present illness, he was not taking any medications. Both his parents were healthy, and did not have a history of major illnesses in the past.

On physical examination he was comfortable. There was no pallor, jaundice or lymphadenopathy. Pulse and blood pressure were normal. The heart and lungs were normal on auscultation, and there were no murmurs or added sounds. On abdominal examination, the spleen was enlarged. Neurological examination did not show any abnormality.

His complete blood counts showed a hemoglobin $(\mathrm{Hb})$ level of $11.6 \mathrm{~g} / \mathrm{dL}$, total leucocyte count $63.0 \times 10^{9} / \mathrm{L}$ and platelet count $172.0 \times 10^{9} / \mathrm{L}$. His abdominal ultrasound revealed splenomegaly. Serum bilirubin, alanine transaminase (ALT), alkaline phosphatase, urea, creatinine, sodium, potassium, uric acid and blood glucose were within normal limits. Routine stool examination did not show any ova or cysts.

His bone marrow examination showed a hyperplastic marrow, reduced megakaryocytes and $45.0 \%$ blast cells. The blast cell population showed strong reactivity $(>95.0 \%)$ with B-cell markers including CD10, CD19, CD20, CD22 and cCD79a. The blasts did not show expression of lymphoid $\mathrm{T}$ markers (CD3, 5 and 7) and myeloid markers (CD 13, 33 and cMPO). The immunophenotype was consistent with common precursor B-lymphoblastic leukemia. Cytogenetic analysis revealed the following karyotype: 45,XY,der(9)t $(9 ; 12)(\mathrm{p} 24 ; \mathrm{q} 12),-12$ [04]/46,XY[16] (Figure 1).This breakpoint includes rearrangement of JAK2 (Janus kinase 2) at 9(p24) loci and CPNE 8 (Copine-8) at 12(q12) genes with a loss of the 12(p) region. Protein product of JAK2 is tyrosine kinase that is involved in signal transduction. Prognosis related to this protein product is highly variable and allogeneic stem cell transplantation may be the only curative treatment. Copine- 8 is a member of the copines. Copines are highly conserved, widely expressed, calcium-dependent membrane binding proteins. They may have a role in membrane trafficking and mediate cellular processes by conferring calcium regulation to various signaling pathways. Copine- 8 is strongly expressed in brain, heart, and prostate, while its expression low in most other tissues. A novel translocation of $12 \mathrm{p} 13$ region in adult

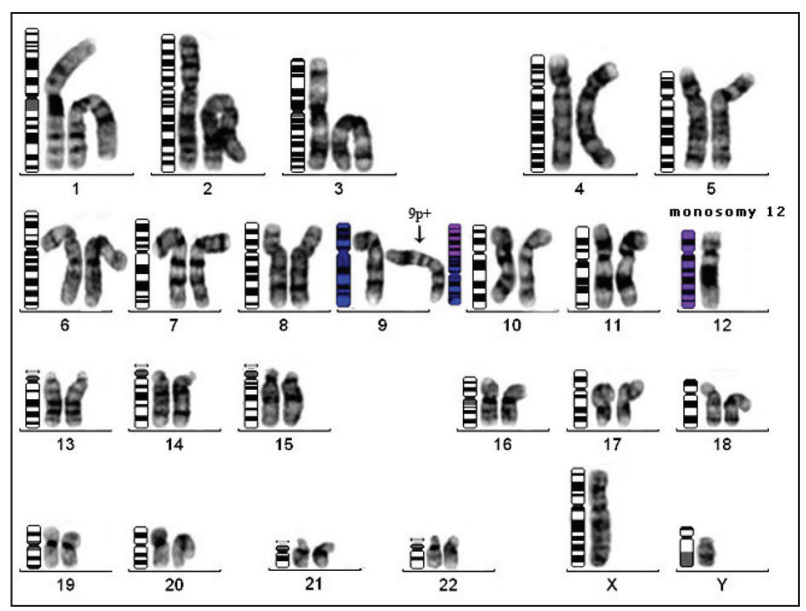

Figure 1. The unique cytogenetic abnormality: 45,XY,der(9)t(9;12)(p24;q12),-12.

B-lymphoblastic leukemia with sever eosinophilia was reported in 2009 [2], while only three case have been reported within the 9p24 region on a different chromosome [3]. A novel case of $\operatorname{dic}(9 ; 12)$ involving the $\operatorname{der}(9) \mathrm{t}(9 ; 22)$ in the Philadelphia chromosome positive common B-cell ALL is unique [4], while only a single case is known of the homozygous deletion of the 9p21 locus corresponding to the pl6 gene in ALL with eosinophilia [5].

The proband was started on induction therapy based on UK ALL XII consisting of a four drugs regimen, which included Inj (injectable) vincristine; Inj daunorubicin; tab (tablets) prednisolone and Inj asparaginase. However, he failed to achieve remission after induction therapy.

\section{CONCLUSIONS}

Adult B-ALL with hyperplastic marrow and $40.0 \%$ blast cells having a cytogenetic abnormality, 45,XY,der(9) $\mathrm{t}(9 ; 12)(\mathrm{p} 24 ; \mathrm{q} 12),-12$ is a distinct entity with poor response to chemotherapy and a bad prognosis. Individuals with this disease should be referred for bone marrow transplantation from a matched sibling or unrelated donor, for best chances of disease-free survival.

\section{REFERENCES}

1. Swerdlow SH, Campo E, Harris NL, Jaffe ES, Stein H, Pileri S. The 2008 World Health Organisation classification of lymphoid neoplasms 
and beyond: evolving concepts and practical applications. WHO Classification of Tumours of Haematopoietic and Lymphoid Tissues, 4th ed. Lyon: IARC Press. 2008: 109-138.

2. Bhatti FA, Hussain I, Muhammad ZA. Adult B lymphoblastic leukaemia/lymphoma with hypodiploidy (-9) and a novel chromosomal translocation $\mathrm{t}(7 ; 12)(\mathrm{q} 22 ; \mathrm{p} 13)$ presenting with severe eosinophilia - case report and review of literature. J Hematol Oncol. 2009; 2(1): 26-31.

3. Atlas of Genetics and Cytogenetics in Oncology and Haematology online, 1998 (http://atlasgeneticsoncol ogy.org/).
4. Bargetzi MJ, Mühlematter D, Tichelli A, Jotterand M, Wernli M. Dicentric translocation $(9 ; 12)$ presenting as refractory Philadelphia chromosomepositive acute B-cell lymphoblastic leukemia. Cancer Genet Cytogenet. 1999; 113(1): 90-92.

5. D'Angelo G, Hotz AM, Todeschin P. Acute lymphoblastic leukemia with hypereosinophilia and 9p21 deletion: case report and review of literature. Lab Hematol. 2008; 14(1): 7-9. 